\title{
TEMPO DE RESISTÊNCIA EM ESTEIRA: ATLETISMO
}

\author{
Kiss, Maria Augusta P.D. Molin* \\ Vallejo Cuellar, Lisímaco** \\ Machida, Junko*** \\ Carvalho, Paulo Roberto de ${ }^{\star \star \star \star}$

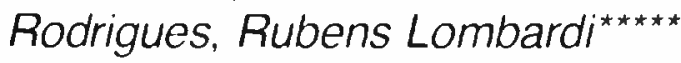

\begin{abstract}
RESUMO
O acompanhamento de treinamento tem sido feito principalmente pela evoluçăo do consumo máximo de oxigênio: contudo ele tem se mostrado insuficiente, pois depois de algum tempo ele se estatiliza mas o desempenho ainda melhora. Com finalidade de seguir esportistas de nivel paulista e/ou brasileiro, por várias temporadas, experimentamos testes de tempo de resistencia a diferentes velocidades, de acordo com os melhores tempos de competicōes de corridas em

$I(n=3)$ arremessadores, examinados a velocidade de $15,6 \mathrm{~km} / \mathrm{h} ; \mathrm{G} \|(n=5)$ fundistas, examinados a velocidade de $18 \mathrm{~km} / \mathrm{h}$ e $G$ III $(n=5)$ velocistas, velocidade de $21.6 \mathrm{~km} / \mathrm{h}$. o grupo $G N(n=8)$, velocidade 16.8 $\mathrm{km} / \mathrm{h}$. A evolução $\mathrm{VO}_{2}$ máx. do primeiro grupo foi significativa. o que não não ocorreu nos demais; houve aumento do tempo de resistencia em esteira entre a orimeira e a terceira época de medida em todos os subgrupos.
\end{abstract} pista. Foram esludados 3 grupos de sexo masculino $G$ nhag e Sjodin, 1985) mas também a resistência em trabalhos intensos, quer em cicloergômetro (Moritana et alii, 1981) quer em esteira rolante (Hugson et alii, 1984); estes dois últimos trabalhos procuram determinar, para cada individuo, uma constante aeróbica e outra anaeróbica.

Nossa preocupação, há algum tempo, tem sido a procura de testes que consigam quantificar a evolução do treinamento de esportistas, através de várias temporadas; por isso, trabalhamos, desde 1974 (Kiss et alii, 1978; Vallejo Cuellar, 1988) corr testes de resistência em esteira, em velocidades relativamente elevadas, com diferentes modalidades esportivas.

Neste trabalho procuramos determinar quais variáveis nos mostram a evolução de grupos de elite, praticantes de atletismo, da Cidade e do Estado de São Paulo.

Professor Livre-Docente das disciplinas Medidas e Avaliação da Educaçāo Fisica e Avaliação em Educação Fisica I e II, da EEF-USP

** Pós-Graduando em Educação Física na EEF-USP

** Auxiliar de Ensino da disciplina de Medidas e Avaliaçāo da Educaçāo Física, da EEF-USP

**** Auxiliar de Ensino da disciplina Crescimento e Desenvolvimento Humano, da EEF-USP

***** Professor Assistente Doutor da disciplina Socorros de Urgência, da EEF-USP 


\section{CASUÍSTICA E MÉTODO}

Foram estudadas duas amostras de esportistas do sexo masculino, praticantes das diferentes especialidades de atletismo; a primeira era constituida por todos os atletas do "Projeto Adote um Atleta" da Prefeitura de São Paulo, que praticavam atletismo em 1976 e foram examinados durante esse ano no Laboratório de Avaliação e Condicionamento Físico da Escola de Educação Física da Universidade de São Paulo, sendo em número de treze. Os esportistas da segunda amostra pertenciam em 1985 e 1986 ao "Projeto Futuro" da Coordenadoria de Esportes do Estado de São Paulo, sendo em número de oito.

A primeira amostra foi subdividida em três grupos em funçāo da especialidade de cada atleta e da velocidade utilizada na esteira rolante.

Inicialmente foram feitas as medidas antopométricas de rotina, seguidas de um teste submáximo em cicloergômetro eletromagnético, utilizando-se o nomograma de Astrand-Ryhming (1954) para cálculo do consumo máximo de oxigênio.

Os cálculos iniciais para a velocidade da esteira para o teste de resistência (TR) foram baseados nos tempos de percurso (e portanto nas respectivas médias de velocidade em campo) dos atletas examinados. Assim, por exemplo, o atleta L.D.S.M. fazia os $3.000 \mathrm{~m}$ em $9^{\prime} 41$, portanto com a velocidade média de $18,59 \mathrm{Km} / \mathrm{h}$, tendo sido testado a $18 \mathrm{Km} / \mathrm{h}$; para comparação na mesma época: o campeāo mundial da prova tinha velocidade média de $24,21 \mathrm{Km} / \mathrm{h}$.

Os três grupos da primeira amostra foram: $\mathrm{G} \mid(n=3)$, constituido por arremessadores, examinados a velocidade de $15,6 \mathrm{Km} / \mathrm{h} ; \mathrm{G} \|(\mathrm{n}=5)$ fundistas e meio fundistas, examinados a velocidade $18 \mathrm{Km} / \mathrm{h}$ e G III $(n=5)$ composto por velocistas e examinados a $21,6 \mathrm{Km} / \mathrm{h}$.

Os atletas do "Projeto Futuro" constituiam o G IV e foram todos examinados na mesma velocidade na esteira rolante, qual seja $16,8 \mathrm{Km} / \mathrm{h}$.

No "Projeto Adote" os exames foram realizados no início, meio e fim do ano de 1976; os atletas do "Projeto Futuro" foram examinados em duas épocas: março de 1985 e março de 1986; para mais fácil localização na tabela estas medidas foram colocadas junto com a correspondente em época do "Projeto Adote"

Foi medido, em cada individuo e em cada fase do treinamento, o tempo máximo, até exaustão, tolerado nas respectivas velocidades: 0 conceito de exaustão foi clínico, manifestado por palidez, cianose e início de incoordenaçāo motora; o atleta desconhecia o tempo que estava fazendo.

A análise de variância a um fator com ou sem dados repetidos, com nivel de significância de $5 \%$, foi utilizada como ferramenta estatistica fundamental; no Grupo IV utilizamos o test t de Student, para dados correlacionados.

\section{RESULTADOS}

Os valores de peso e estatura dos 4 grupos, no início de cada trabalho, constam da Tabela I. Houve aumento significativo do peso em todos os grupos, contudo o mesmo nāo ocorreu com a estatura, que apresentou diferença significativa apenas no G IV.

O consumo máximo de oxigênio $\left(\mathrm{VO}_{2}\right.$ máx) não apresentou evolução significativa em nenhum grupo, quer no "Projeto Adote um Atleta" quer no "Projeto Futuro" (Tabela II).

\section{TABELA I}

Idade, estatura, peso e número de individuos dos Grupos G I, G II e G III do "Projeto Adote um Atleta" e G IV do "Projeto Futuro": valores médios ( $x$ ) e desvios padrões (s), no início dos trabalhos.

\begin{tabular}{|c|c|c|c|c|c|c|c|}
\hline & \multirow{2}{*}{$\frac{\text { Grupo }}{\mathrm{n}}$} & \multicolumn{2}{|c|}{$\begin{array}{l}\text { Idade } \\
\text { (anos) }\end{array}$} & \multicolumn{2}{|c|}{$\begin{array}{c}\text { Estatura } \\
\text { (cm) }\end{array}$} & \multicolumn{2}{|c|}{$\begin{array}{l}\text { Peso } \\
\text { (kg) }\end{array}$} \\
\hline & & $\bar{x}$ & $\mathbf{s}$ & $\bar{x}$ & $s$ & $\bar{x}$ & s \\
\hline G I & 3 & 16,7 & 0,58 & 180,30 & 5,62 & 76,76 & 10,47 \\
\hline G II & 5 & 16,6 & 1,14 & 170,94 & 8,99 & 60,90 & 9,25 \\
\hline G III & 5 & 16,8 & 0,41 & 180,20 & 10,30 & 67,70 & 8,89 \\
\hline G IV & 8 & 16,8 & 0,30 & 174,30 & 3,27 & 62,80 & 3,48 \\
\hline
\end{tabular}


Consumo máximo de oxigênio (VO2 máx em 1/min e em $\mathrm{ml} / \mathrm{Kg} / \mathrm{min}$ : evolução das medidas nos diferentes grupos valores médios $(x)$ e desvio padrão (s): resultado da análise de variância para dados repetidos, a um fator; ns - não significativo.

\begin{tabular}{|c|c|c|c|c|c|c|c|}
\hline \multirow{3}{*}{ Grupo } & & \multicolumn{6}{|c|}{ VO$_{2}$ Máx } \\
\hline & & \multicolumn{3}{|c|}{$1 / \min$} & \multicolumn{3}{|c|}{$\mathrm{ml} / \mathrm{Kg} / \mathrm{min}$} \\
\hline & & 1 & 2 & 3 & 1 & 2 & 3 \\
\hline \multirow[t]{2}{*}{ GI } & $\bar{x}$ & 3,02 & 3,82 & 3,92 & 39,7 & 48,0 & 52,7 \\
\hline & $s$ & 0,69 & 0,69 & 0,78 & 7,5 & 10,8 & 12,5 \\
\hline \multirow[t]{2}{*}{ G II } & $\bar{x}$ & 3,35 & 3,56 & 3,56 & 54,4 & 58,0 & 55,0 \\
\hline & $\mathrm{s}$ & 0,26 & 0,45 & 0,59 & 11,6 & 8,5 & 4,6 \\
\hline \multirow[t]{2}{*}{ G III } & $\bar{x}$ & 2,95 & 3,15 & 3,15 & 44,2 & 48,0 & 45,4 \\
\hline & $\mathbf{s}$ & 0,24 & 0,50 & 0,70 & 9,1 & 13,2 & 9,9 \\
\hline \multirow[t]{2}{*}{ G IV } & $\bar{x}$ & 3,72 & & 3,71 & 59,9 & & 57,9 \\
\hline & $\mathbf{s}$ & 0,81 & & 1,06 & 12,7 & & 15,1 \\
\hline
\end{tabular}

NOTA :o reteste do Grupo G IV foi colocado em conjunto com as terceiras medidas dos demais grupos pela semelhança dos intervalos de tempo entre as coletas.
Em relação ao tempo de resistência em esteira rolante houve aumento significativo entre a primeira e a terceira medidas, nos grupos G II, G III e G IV; no grupo G III houve diferença também entre a segunda e a terceira medidas (Tabela III).

TABELA III

Tempo de resistência em esteira rolante, a $15,6 \mathrm{~km} /$ - no Grupo G I; a $18 \mathrm{~km} / \mathrm{h}$, no G II, a 21,6 km/h no G III e a $16,8 \mathrm{~km} / \mathrm{h}$ no $G \mathrm{IV}$; valores médios $(x)$ e desvio pacirão (s) nas diferentes épocas de teste: resultados da análise de variância para dados repetidos (ns nāo significativo; ' significativo ao nivel de $5 \%$ ).

\begin{tabular}{|c|c|c|c|c|c|}
\hline \multirow{2}{*}{ Grupo } & & \multicolumn{4}{|c|}{ Tempo de resistência (s) } \\
\hline & & 1 & 2 & 3 & $\mathbf{F}$ \\
\hline G I & $\begin{array}{l}\bar{x} \\
\mathrm{~s}\end{array}$ & $\begin{array}{l}88,5 \\
16,3\end{array}$ & $\begin{array}{r}103,7 \\
11,7\end{array}$ & $\begin{array}{l}268,3 \\
102,6\end{array}$ & ns \\
\hline$G \|$ & $\begin{array}{l}\dot{x} \\
s\end{array}$ & $\begin{array}{r}101,4 \\
13,2\end{array}$ & $\begin{array}{l}170,8 \\
106,3\end{array}$ & $\begin{array}{l}345,4 \\
246,9\end{array}$ & * \\
\hline G III & $\begin{array}{l}\dot{x} \\
s\end{array}$ & $\begin{array}{l}56,8 \\
26,2\end{array}$ & $\begin{array}{l}48,8 \\
26,5\end{array}$ & $\begin{array}{r}117,8 \\
50,7\end{array}$ & * \\
\hline G IV & $\begin{array}{l}\bar{x} \\
s\end{array}$ & $\begin{array}{l}451,3 \\
332,9\end{array}$ & & $\begin{array}{r}1041,1 \\
692,5\end{array}$ & * \\
\hline
\end{tabular}




\section{COMENTÁRIOS}

Os atletas do Projeto "Adote um Atleta" tiveram aumento de peso apenas no Grupo G I; nenhum dos grupos apresentou desenvolvimento da variável aeróbica, expressa pelo $\mathrm{VO}_{2}$ máx, em $\mathrm{ml} / \mathrm{kg} / \mathrm{min}$, discordando não só dos trabalhos de escolares de Daniels et alii (1978), mas também do encontrado em corredores por Kobayashi et alii (1978).

Interessante frizar que o tempo de resistência em esteira tendeu a melhorar no grupo G II já na primeira fase do treinamento, mas a diferença não foi significativa; o grupo G III, que teve velocidade de $21,6 \mathrm{~km} / \mathrm{h}$, apresentou estabilização dos resultados entre o primeiro e segundo testes, mas aumento significativo no terceiro.
Os atletas do "Projeto Futuro" (G IV) também tiveram melhoria significativa no tempo de resistência em esteira, mas não no $\mathrm{VO}_{2}$ máx.

A melhoria do tempo de resistência provavelmente deveu-se não só a uma maior eficiência (Svednhag \& Sjodim, 1985), mas também a uma maior fração do $\mathrm{VO}_{2}$ máx utilizada sem produção de lactato (Förenbach et alii, 1987), somados a uma maior capacidade anaeróbica.

Dessa forma sugerimos que o acompanhamento em atletismo seja feito por teste de esteira e não por cicloergômetro, levando-se em conta para futuro, dois testes de tempo de resistência em velocidades elevadas de esteira, que permitam quantificar objetiva e individualmente os componentes aeróbico e anaeróbico.

\section{REFERÊNCIAS BIBLIOGRÁFICAS}

ASTRAND, P.O. \& RYHMING, I. A nomogram for calculation of aerobic capacity (Physical Fitness) from pulse rate during submaximal work. J. Appl. Physiol., Bethesda, 7: 212-21, 1954.

COSTIL, D.; THOMASON, H. \& ROBERTS, E. Fractional utilization of the aerobic capacity during distance running. Med. Sci. Sports, Madison, 5(4): 248-52, 1973.

DANIELS, J. et alii. Differences and changes in $\mathrm{VO}_{2}$ max among young runners $10-18$ years of age. Med. Sci. Sports, 10(3): 200-3, 1978.

FÖHRENBACH, R.; MADER, A.B. \& HOLLMANN, $W$. Determination of endurance capacity and prediction of exercice intensities for training and competition in marathon runners. Int. J. Sports Med., Stuttgart, 8(1): 11-8, 1987.

GLESSER M.A. \& VOGEL, J.A. Endurance capacity for prolonged exercise on the bicycle ergometer. In: McLELLAN, J.M.B.; SKINNER, J.S. Submaximal endurance performance related to the ventilation thresholds. Can. J. Appl. Sports Sci., Toronto, 10(2): 81-7, 1985.
HECK, G. et alii. Justification of the $4 \mathrm{mmol} / \mathrm{l}$ lactato thresholds. Int. J. Sports Med., Stuttgart, 6(2): 117-30, 1985.

HUGHSON, R.L. et alii. A high velocity treadmill running test to assess endurance running potencial. Int. J. Sports Med., Stuttgart, 5(1): 23-5, 1984.

KISS, M.A.P.D.M. Introduçāo a ciência do esporte. São Paulo, EEFUSP, 1978.

KISS, M.A.P.D.M. et alii. Maximal time of endurance et high velocity in treadmill and aerobic condition in judo. In: WORLD CONG SPORTS MEDICINE, 21, Brasilia, 1978, p. 247.

KISS, M.A.P.D.M. Avaliaçāo em Educação Física: aspectos biológicos e educacionais. São Paulo, Manole, 1987.

KISS, M.A.P.D.M. et alii. Limiar de lactato no teste de endurance escalonado em crianças e adolescentes. In: SIMPÓSIO INTERNACIONAL DE MEDICINA DESPORTIVA, $2^{\circ}$, Rio de Janeiro, 1987, p.12. 
KOHAYASHI, K. et alii. Aerobic power as related to body growth and training in japanese boys: a longitudinal study. J. Appl. Physiology, 44: 666-72, 1978.

MCLELLAN, T.M. \& SKINNER, J.S. Submaximal endurance performance related to the ventilation thresholds. Can. J. Appl. Sport Sci., Toronto, 10(2): $81-7,1985$.

MORITAMI, T et alii. Critical power as a measure of physical work capacity and anaerobic threshold. In: HUGSON, R.L.; OROK, C.J. \& STAUDT, L.E. A high treadmill running to assess endurance running potencial. Int. J. Sport Med., 5(1): 23-5, 1984.
SVEDENHAG, J. \& SJÖDIN, B. Physiological characteristics of elite male runners in and off seasun. Can. J. Spuil Sci., Toronto, 10(3): $127-33,1985$.

VALLejo Cuellar, L. Análise da Capacidade aeróbica em atletas adolescentes. Tese de Mestrado em Educação Física. Sāo Paulo, 1988.

WILEY, J.P. \& RHODES, E.L. The relationship of individual anaerobic thresholds to total, lactic and oxigen debts after a set threadmill run. Can. J. Appl. Sport Sci. Toronto, 11(1): 37-41, 1986. 